\title{
Tau Ubiquitination in Alzheimer's Disease
}

\author{
Longfei Li ${ }^{1+}$, Yanli Jiang ${ }^{1+}$, Jian-Zhi Wang ${ }^{1,2,3}$, Rong Liu ${ }^{1}$ and Xiaochuan Wang ${ }^{1,2,3 *}$ \\ ${ }^{1}$ Key Laboratory of Education Ministry/Hubei Province of China for Neurological Disorders, Department of Pathophysiology, \\ School of Basic Medicine, Tongji Medical College, Huazhong University of Science and Technology, Wuhan, China, \\ ${ }^{2}$ Co-innovation Center of Neuroregeneration, Nantong University, Nantong, China, ${ }^{3}$ Department of Pathology and \\ Pathophysiology, School of Medicine, Jianghan University, Wuhan, China
}

OPEN ACCESS

Edited by:

Liyong Wu,

Capital Medical University, China

Reviewed by:

Yingjun Zhao,

Xiamen University, China

Benjamin Combs,

Michigan State University,

United States

Jianhua Shi,

Harvard Medical School,

United States

Chunling Dai,

Institute for Basic Research in Developmental Disabilities (IBR),

United States

Yongku Cho,

University of Connecticut,

United States

*Correspondence:

Xiaochuan Wang

wxch@mails.tjmu.edu.cn

tThese authors have contributed equally to this work

Specialty section:

This article was submitted to Dementia and Neurodegenerative Diseases,

a section of the journal

Frontiers in Neurology

Received: 30 September 2021 Accepted: 29 December 2021

Published: 08 February 2022

Citation:

Li L, Jiang Y, Wang J-Z, Liu R and Wang $X$ (2022) Tau Ubiquitination in Alzheimer's Disease.

Front. Neurol. 12:786353. doi: 10.3389/fneur.2021.786353
Paired helical filaments (PHFs) from the Alzheimer's disease (AD) brain are highly ubiquitinated and ubiquitination likely plays a vital role in tau filament formation. Whether tau ubiquitination is the causality or consequence of the disease in AD remains elusive. The following questions are worth considering: What does the extent of tau ubiquitination contribute to tau pathology in AD? Does tau ubiquitination influence aggregation or spreading during disease progression? In addition, tau is polyubiquitinated in nerve growth factor-induced PC12 cells and participates in mitogen-activated protein kinase signaling, in addition to its microtubule stabilization function. Therefore, ubiquitination possibly mediates tau signaling under physiological conditions, but tau aggregation in the pathobiology of $A D$. Here, we review the advancements in tau ubiquitination and the potential therapeutic effects of targeting tau ubiquitination to alleviate tau pathology in $A D$.

\section{Keywords: PHF, Alzheimer's disease, tau, NGF, ubiquitination}

\section{INTRODUCTION}

Ubiquitin-positive inclusions are characteristic of neurodegenerative diseases, such as Alzheimer's disease (AD) and frontal temporal lobe degeneration (1-3). Neurofibrillary tangles, mainly consisting of hyperphosphorylated tau, are also ubiquitin and p62 positive in $\mathrm{AD}(4,5)$. In fact, ubiquitin is elevated by many folds in the AD brain determined by immunoassay (1), and the ubiquitination level increases at $\sim 80 \%$ ubiquitylated sites from the altered 800 ubiquitination sites by label-free mass spectrometry (MS)-based proteomic analysis (6). Among the ubiquitination proteins, the microtubule-associated protein tau has the highest number of sites of ubiquitination per protein in $\mathrm{AD}$ (6). In addition, the data from cryoelectron microscopy (EM) and MS of tau filaments from $\mathrm{AD}$ and corticobasal degeneration brain further demonstrate ubiquitination of tau in neurofibrillary tangles, and this posttranslational modification might play a structural role in its fibrillation and fibril heterogeneity (7-9). This review aimed to conclude the influence of tau ubiquitination in the pathobiology of $\mathrm{AD}$ and provide valuable cues for future studies.

\section{TYPE OF TAU UBIQUITINATION IN AD}

The 76-amino acid protein ubiquitin can be transferred to lysine with subjects through the three enzyme cascade steps: ubiquitin activation, conjugation, and ligation by E1, E2, and E3 enzymes in succession (10). The substrate can be either modified by a monomer or a polyubiquitin chain depending on E2s, whereas the substrate specificity is determined by E3 ligases (11). 
In addition to monoubiquitylation, a polyubiquitin chain occurs when another ubiquitin is conjugated to any of the seven lysines or N-terminus on the first ubiquitin by its C-terminal GG amino acid (12). Ubiquitin modification and its signal for cell output and degradation are reviewed in detail (10-14). In this review, these were not specified, and this review only focused on tau ubiquitination in $\mathrm{AD}$.

The microtubule-associated protein tau has 44 lysine residues on the longest 441 isoforms, most of which are located on the proline-rich domain and microtubule-binding domain repeat (MTBR) (15). Tyrosine kinase family proteins, such as fyn and c-src, bind to tau with the PXXP motif in the proline-rich domain and execute its function $(16,17)$, whereas tau promotes microtubule stabilization through the MTBR domain (18). The abundant lysine and arginine make full-length tau $\mathrm{pI}=8.24$, and tau's repeat domain (termed K18) pI = 9.73 (15). Furthermore, 28 tau ubiquitylation sites detected in human $\mathrm{AD}$ brain samples underlie the tau to become the highest number of increased ubiquitination sites per protein, as discussed above (6). Among these sites, K257, K259, K267, K274, K281, K290, K321, K343, K353, K375, and K385 are ubiquitinated by E3 ligase Chip (Figure 1) (7). The most frequently reported sites are Lys254, Lys257, Lys311, Lys317, and Lys353 (6, 8, 19, 20).

To date, K48-, K63-, K6-, K11-, and M1-linked polyubiquitin chains have been verified in tau of paired helical filament (PHF), although the majority of ubiquitination in PHF is in monoubiquitinated form (19-21). K48-linked polyubiquitination is the most common form of tau poly- $\mathrm{Ub}$ and is hypothesized to mediate protein degradation by the proteasome system (19). K63-linked poly-Ub of proteins can serve multiple functions for various proteins, including promoting insoluble inclusion formation (22), signal for autophagy lysosome (13), endocytosis (23), and DNA repair (24). A part of PHF is K63-linked poly Ub-positive, as detected in the $\mathrm{AD}$ brain (25). One study reported that to a lesser extent K6/11 poly-Ub existed in PHF by tandem mass spectrometry assay, and K6-linked poly-Ub may inhibit proteasome activity (20). M1-linked linear poly-Ub, which is involved in neuronal cell death, appears after K48-linked poly-Ub of PHF $(21,26)$. A few studies revealed that tau mono-Ub impaired its microtubule binding (27) and that the N-terminal tau mono-Ub could impede its aggregation by cooperating with the proteasome system (28).

\section{REGULATION OF TAU UBIQUITINATION IN AD}

The identification of the types of tau ubiquitination is accompanied by the recognition of the E1, E2, and E3 enzymes involved in tau ubiquitination. Regarding mono-Ub, axotrophin/MARCH7 containing a RING-variant domain could monoubiquitinate tau combined with E2 UbcH5 and impair its microtubule binding (29). A recent study reported that E2 Ube2W attached mono-Ub to the $\alpha \mathrm{N}$-terminus of tau by recognizing backbone atoms of disordered N-termini (30). In vivo, CHIP modifies tau through both K48- and K63-linked polyubiquitination (31), and tau is also a K63-polyubiquitinated substrate of TRAF6/UbcH7 (32). Furthermore, the ubiquitin elongation enzyme $\mathrm{UBE} 4 \mathrm{~B}$ has been reported to promote tau polyubiquitination sufficiently by cooperating with CHIP, thereby promoting tau degradation (33).

In addition to identifying E3 ligase on tau ubiquitination, other inducers, such as nerve growth factor (NGF), can promote tau ubiquitination through unknown mechanisms. NGF maintains neuronal survival and differentiation in PC12 through its receptor TrkA with the elevation ( $>2$-fold) of ubiquitin conjugate levels; a similar result could be mimicked by a proteasome inhibitor (34). NGF also stimulates tau ubiquitination through K63-linked poly-Ub in neuronal cell differentiation (25), which implies the possibility of the downregulation of the NGF signaling pathway in $\mathrm{AD}$ pathogenesis by impairing normal tau ubiquitination, as the decrease of NGF in the cortex and hippocampus mediates cholinergic neuron degeneration in the basal forebrain at disease onset.

\section{ROLE OF UBIQUITINATION IN TAU TURNOVER}

Six isoforms of tau by alternative slicing are mainly expressed in the central nervous system (CNS) (35). CNS tau is a longlived and natively disordered protein, which has a half-life of $\sim 23$ days. Under physiological conditions, tau is soluble and has a hairpin structure in which the MTBR is buried under the inner layers of the C-terminus and the outer layers of the N-terminus, as detected by fluorescence resonance energy transfer (36). An average of 2-3 mol of phosphate per mole of protein in normal people and 6-8 mol of phosphate per mole of protein in the AD brain indicates that abnormal hyperphosphorylation of tau plays an important role in the pathobiology of AD (37-39). Several studies have already reported how hyperphosphorylation of tau leads to neurotoxicity and epigenetic risk factors, such as trauma, sleeplessness, and less exercise, influencing tau phosphorylation and cognitive function (40). Thus, tau phosphorylation is widely used as a biomarker in cell and animal models in AD studies. Tau phosphorylation at threonine 181 and 217 in the cerebrospinal fluid or plasma have shown their potential as a biomarker for predicting the extent of dementia $(41,42)$. AT8 staining is considered the gold standard for detecting pretangles in immunohistochemistry (IHC), as it is applied by BraaK (43).

Phosphorylation of tau could change its conformation and open its hairpin structure, resulting in the formation of tau oligomers despite the exact mechanisms of oligomer formation being still elusive. Many factors can affect this process, such as extending its $\mathrm{N}$-terminus or $\mathrm{C}$-terminus to protease, resulting in truncation and accelerating tau aggregation, or being recruited to stress granules with its abundant lysine in the proline-rich (PR) and MTBR domains under cell stress (15, 44-47). In 1989-1991, it was reported that ubiquitin levels increased many folds in the $\mathrm{AD}$ brain, and the accumulation of abnormally phosphorylated tau preceded the formation and ubiquitination of neurofibrillary tangle (NFT) $(1,48,49)$. About 2 years later, 


\section{1 maeprqefev medhagtygl gdrkdqggyt mhqdqegdtd aglkesplqt ptedgseepg}

\section{1 setsdakstp taedvtaplv degapgkqaa aqphteipeg ttaeeagigd tpsledeaag}

\section{1 hvtqarmvsk skdgtgsddk kakgadgktk iatprgaapp gqlkgqanatr ipaktppapk}

181 tppssgeppli sgdrsgyssp gspgtpgsrs rtpslptppt replkkvavvr tpplsspssalk

241 srlqtapvpm pdlknvliski gstenllihqp ggglivqiink kldlsnvqsk cgskdnilkhv

301 pgggsvqivy kpvdlslivts licgslgnihh lipgggqvevk selildfkdrv qsligssldni

361 thvpgggnlkk iethlkltfre nalkaktdhga eivylkspvvs gdtsprhlsn vsstgsidmv

\section{1 dspqlatlad evsaslakqg 1}

FIGURE 1 | The sequence of 2N4R-tau with identified ubiquitinated sites (both purple and red, k). The yellow sequence indicates proline-rich domain, whereas cyan indicates microtubule-binding repeat domain. Chip ubiquitinates tau at sites shown by red color.

Yasuo Ihara et al. purified high-molecular weight (HMW) Ub (-) PHF and ubiquitin (+) PHF from PHF tau and found that the amino-terminal portion of tau was truncated in $\mathrm{Ub}$ (-) PHF and to a greater extent in HMW ubiquitin (+) $\mathrm{PHF}$ (19). A more detailed study was conducted several years later, using tau oligomer-specific antibody T22 in combination with pT231, AT8, and ubiquitin antibodies. Kayed et al. proposed a more specific sequence of tangle formation with minor revision of previous results: tau phosphorylation at the T231 site (stage 0); tau oligomer initiation and formation with pT231 (stage 1) and intraneuronal NFT (iNFT) containing mixed oligomer, protofilament, and filament (stage 2); and neuronal death and the formation of ghost tangle with T22 negativity and pT231 positivity, referred to as extraneuronal NFT (eNFT) (stage 3). Besides the AT8 property of the staining pretangle, most eNFTs are AT8 positive. By comparing the time sequence of ubiquitination and tau oligomerization, they suggest that ubiquitination does not appear at the initial stage of tau oligomerization, but it occurs on iNFT (stage 2) followed by high ubiquitination on eNFT (50). The above studies suggest that abnormal phosphorylation likely mediates tau oligomerization (51). As the soluble tau oligomer grows and is modified by truncation and ubiquitination, it gradually matures to fibrillary tangles and is insoluble with tangle formation. This transformation suggests that ubiquitination probably promotes insoluble filament formation from soluble tau aggregates $(8,52)$.

To better understand the effect of ubiquitination on tau aggregation, Kah-Leong Lim explored the contribution of K48- and K63-linked poly-Ub and monoubiquitination to the biogenesis of inclusions in cell models and found that K63-linked poly-Ub usually promoted inclusion formation, such as tau and sod1 aggregates and their clearance by autophagy (22). In fact, CHIP-mediated K48-/K63-linked poly-Ub promotes insoluble tau formation, whereas HSP70 suppresses it (31). Moreover, CHIP knockout mice show accumulation of nonaggregated, ubiquitin-negative, and hyperphosphorylated tau, demonstrating that CHIP mediates ubiquitin-dependent tau degradation and poly-Ub of tau by CHIP accelerating the formation of insoluble filaments (53). CHIP is an E3 ligase of phosphorylated tau. CHIP mediates tau ubiquitination at K267, K290, K343, and K353 sites, but additional K257, K259, K274, K281, K321, K375, and K385 are ubiquitinated by CHIP after tau is phosphorylated by glycogen synthase kinase-3 $\beta$. Prolonged phosphorylated tau ubiquitination promotes tau aggregation (7). In another study, traf6-mediated K63-linked poly-Ub also showed a similar effect on tau aggregation (32).

Contrary to these studies, Munari et al. recently studied the aggregation rate of $\mathrm{K} 18$ and $\mathrm{Ub}-\mathrm{K} 18$ in vitro by applying semisynthetic and enzyme-mediated conjugate methods (27). They used CHIP/UBC13 rather than E2 UBCH5 to perform K18 (fragment of 4R-tau) ubiquitination in the tube, which resulted in tau mono-Ub rather than poly-Ub. Unexpectedly, these modifications make K18 lose the ability to convert into amyloid fibrillary structures. Meanwhile, conjugating the mono-Ub to a specific site in K18 by the semisynthetic method would slow down the aggregation but permit the aggregation formation at Lys254 and mostly at Lys353. Lys 311 ubiquitination might follow the formation of NFT and determine fibril subtypes because it is located within the core region of tau for the formation of NFT, which will hinder the aggregation (27).

The N-terminal connected mono-Ub of K18 by UBE2W also slows down the K18 aggregated kinetics and targets oligomers formed by N-mono-Ub-modified K18 to proteasomal degradation compared to unmodified K18 (28). These studies suggest that ubiquitination influences tau aggregation in vitro. However, it does not consider which ubiquitination usually occurs later than tau phosphorylation and oligomerization, as discussed above, in pathological conditions, although tau monomer needs ubiquitination to be degraded by proteasomes in physiological status. 
Proteasomes have recently been shown to fragment tau filaments into oligomers in vitro and degrade oligomers formed from N-terminal mono-Ub-linked K18 (54). Regardless of whether CHIP or Traf6 mediates tau ubiquitination, both have been demonstrated to promote tau degradation by the proteasome. In contrast, tau oligomers can impair proteasomes and decrease proteasome activity in AD brains (55). Therefore, the following should be taken into consideration: (1) Elevation of ubiquitin several folds in the $\mathrm{AD}$ brain and ubiquitinpositive inclusions being common in neurodegeneration; (2) the spatiotemporal order of tau phosphorylation, oligomerization, and ubiquitination during the formation of PHF; (3) loss of proteasome activity in the $\mathrm{AD}$ brain (56); and (4) the neurotoxicity of oligomer tau rather than $\operatorname{PHF}(57,58)$. This can outline the following vicious circle model in cells to explain the roles of ubiquitination in the regulation of tau degradation and aggregation: (1) protein folding-refolding machinery (CHIP/HSP70/90) supervises the folding status of tau (normal tau stage); (2) once tau is abnormally phosphorylated with conformational changes and forms oligomers, a protein quality control system will observe these changes and initiate ubiquitination of the misfolded protein, which promotes insoluble tau formation and its degradation by the proteasome (oligomer tau stage); and (3) with consistent oligomer tau stages, the proteasome is overloaded and cracked; however, without the normal function of the proteasome, these insoluble aggregates accumulate over time and mature into filaments with further ubiquitination, phosphorylation, and truncation ( $\mathrm{Ub}+\mathrm{PHF})$ (NFT stage).

\section{IS TAU UBIQUITINATION BENEFICIAL TO NEURON SURVIVAL IN AD?}

Despite evidence suggesting that tau ubiquitination is involved in tau pathology, including tau mislocated to dendrites or promoting tau aggregation $(7,59)$, there is no direct evidence showing that tau ubiquitination is neurotoxic. However, as the primary function of ubiquitination is to mediate protein degradation, tau ubiquitination is likely nontoxic. Hyperphosphorylated soluble and oligomer tau is hypothesized to have the highest seeding activity and is the most toxic tau species among tau monomers, oligomers, and multimers (58, 6063). As discussed above, ubiquitination increases tau insolubility and occurs at a relatively late stage during the formation of NFTs. Thus, soluble and toxic tau oligomers are transformed into insoluble and less toxic NFTs after hyperubiquitination. In fact, neurons bearing NFTs can survive for many years $(64,65)$. Soluble tau, rather than insoluble NFTs, results in neuronal loss and cognitive dysfunction in a model of tauopathy (57). Based on this evidence, ubiquitination likely protects neurons from the toxicity of oligomer tau by promoting insoluble NFT formation, which is consistent with the concept that polyubiquitination promotes tau aggregation. In contrast, the prion-like activity of pathologic tau decreases with longevity, despite an increase in insoluble tau in $\mathrm{AD}$, and the seeding activity of oligomer tau is higher than that of sarkosyl-insoluble tau. Thus, we can hypothesize that ubiquitination will decrease the seeding and toxic activity of oligomer tau by promoting insoluble NFT formation, but this needs to be proven by experiments. Under physiological conditions, the tau monomer or oligomer is ubiquitinated and mediated to the proteasome for degradation. However, as $\mathrm{AD}$ progresses, proteasome impairment results in the accumulation of ubiquitinated proteins. The dysfunction of this process might contribute to the formation of NFTs and result in neurofibrillary neurodegeneration (Figure 2).

\section{ALZHEIMER'S DISEASE THERAPEUTIC APPROACHES BASED ON TAU UBIQUITINATION}

As discussed above, ubiquitination is good for tau degradation or promotes insoluble and less-toxic aggregate formation from soluble toxic oligomers under physiological or pathological conditions. Therefore, targeting tau ubiquitination should be an effective approach for the treatment of AD. In fact, the benefits of tau ubiquitination are supported by the fact that upregulation of tau ubiquitination through biochemical methods can reduce tau levels and improve impaired memory in an $\mathrm{AD}$ mouse model in vivo. Using a chimera with tau binding domain and E3 ligasebinding moieties, TH006 can specifically induce endogenous tau degradation by promoting tau polyubiquitination (66). A similar chimera, C004019, with the same routine has similar effects and improves cognitive function in an $\mathrm{AD}$ mouse model (67). In addition, mislocated tau in the postsynaptic fraction is hyperphosphorylated and ubiquitinated, and tau from the postsynaptic fraction is a seeding component. Promoting tau degradation in this area by elevating proteasome activity by stimulating the PAC1 receptor-mediated cyclic adenosine monophosphate/protein kinase A (cAMP/PKA) pathway has been shown to improve impaired cognitive performance in rTg4510 mice (67). Another direct evidence is from the Drosophila transgenic model. Overexpression of UBE4B, an E4 ubiquitin elongation enzyme, promotes tau ubiquitination and degradation and alleviates eye neurodegeneration, which is blocked by knockout of the E3 ligase CHIP. Furthermore, this enzyme can reduce tau oligomer levels in a tau transgenic model (33). Based on this evidence, we can conclude that promoting tau ubiquitination or elevating proteasome activity promotes tau degradation and improves tau-induced neurodegeneration and cognitive dysfunction in vivo, despite the fact that hyperpolyubiquitination promotes tau aggregation in vitro. Therefore, promoting tau ubiquitination along with elevated proteasome activity might provide an effective avenue for $\mathrm{AD}$ treatment by reducing tau protein levels.

\section{CONCLUSION AND REMARKS}

Ubiquitination can induce ubiquitin-positive and ubiquitininsoluble tau formation, which could mediate protein degradation. Polyubiquitination protects cells from the toxicity of the soluble oligomer tau. However, these ubiquitin-positive and insoluble tau aggregates accumulate in cells if the degradation 


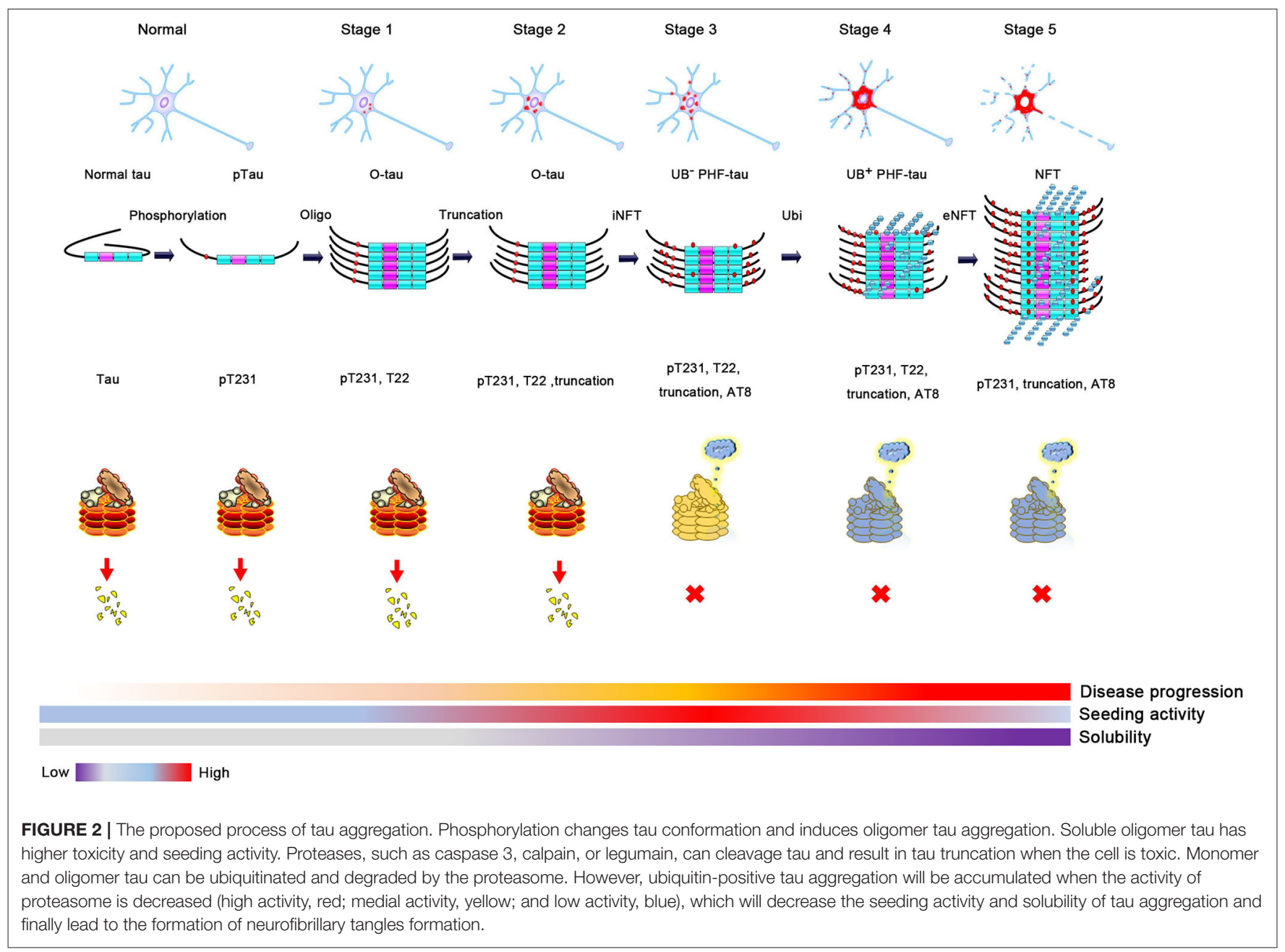

system is damaged under disease conditions. Ubiquitination of tau is good for cell survival, as many studies aimed to induce tau ubiquitination artificially, having beneficial effects on improving abnormal behavior in an $\mathrm{AD}$ mouse model. In addition, the genetic upregulation of E3 or E4 ubiquitin ligases is also useful. Among them, CHIP is the most well-studied E3 ligase for tau ubiquitination. CHIP mainly ubiquitinates phosphorylated tau and promotes the formation and degradation of insoluble tau. Deletion of CHIP in P301L mice led to soluble and phosphorylated tau accumulation. Furthermore, the sites in tau ubiquitinated by CHIP have recently been identified in vitro (7). These studies strongly suggest that ubiquitination promotes NFT formation. In other words, NFT formation might be a consequence of tau ubiquitination without being efficiently degraded by the proteasome. Thus, promoting proteasome activity degrades these ubiquitin-positive tau aggregates. In contrast, reducing tau ubiquitination by inactivating E3 ligase results in soluble tau accumulation, which is a disaster for cell survival. Because HMW tau oligomers in the soluble fraction have higher seeding activity and are the most toxic, it is worthwhile to study the effect of ubiquitination on oligomer tau seeding activity and neurotoxic ability. As phosphorylation or acetylation of tau has been widely explored, the role of ubiquitination in tau-mediated neurodegeneration is believed to be the next hot topic in the future.

\section{AUTHOR CONTRIBUTIONS}

XW framed and reviewed the manuscript. LL and YJ organized the literature and wrote the manuscript. J-ZW and RL analyzed and discussed the manuscript. All the authors read and approved the final version of the manuscript.

\section{FUNDING}

This review article was supported by grants from the National Natural Science Foundation of China (92049107, 31771114, and 31929002), the Innovative Research Groups of the National Natural Science Foundation of China (81721005), and the Academic Frontier Youth Team Project to XW from the Huazhong University of Science and Technology.

\section{ACKNOWLEDGMENTS}

We would like to thank Editage (www.editage.com) for English language editing. 


\section{REFERENCES}

1. Wang GP, Khatoon S, Iqbal K, Grundke-Iqbal I. Brain ubiquitin is markedly elevated in Alzheimer disease. Brain Res. (1991) 566:14651. doi: 10.1016/0006-8993(91)91692-T

2. Neumann M, Sampathu DM, Kwong LK, Truax AC, Micsenyi MC, Chou TT, et al. Ubiquitinated TDP-43 in frontotemporal lobar degeneration and amyotrophic lateral sclerosis. Science. (2006) 314:130-3. doi: $10.1126 /$ science. 1134108

3. Iqbal K, Grundke-Iqbal I. Ubiquitination and abnormal phosphorylation of paired helical filaments in Alzheimer's disease. Mol Neurobiol. (1991) 5:399-410. doi: 10.1007/BF02935561

4. Grundke-Iqbal I, Iqbal K, Quinlan M, Tung YC, Zaidi MS, Wisniewski HM. Microtubule-associated protein tau. A component of Alzheimer paired helical filaments. J Biol Chem. (1986) 261:6084-9. doi: 10.1016/S0021-9258(17)38495-8

5. Grundke-Iqbal I, Iqbal K, Tung YC, Quinlan M, Wisniewski HM, Binder LI. Abnormal phosphorylation of the microtubule-associated protein tau (tau) in Alzheimer cytoskeletal pathology. Proc Natl Acad Sci U S A. (1986) 83:4913-7. doi: 10.1073/pnas.83.13.4913

6. Abreha MH, Dammer EB, Ping L, Zhang T, Duong DM, Gearing M, et al. Quantitative analysis of the brain ubiquitylome in alzheimer's disease. Proteomics. (2018) 18:e1800108. doi: 10.1002/pmic.201800108

7. Kim JH, Lee J, Choi WH, Park S, Park SH, Lee JH, et al. CHIP-mediated hyperubiquitylation of tau promotes its self-assembly into the insoluble tau filaments. Chem Sci. (2021) 12:5599-610. doi: 10.1039/D1SC00586C

8. Arakhamia T, Lee CE, Carlomagno Y, Duong DM, Kundinger SR, Wang $\mathrm{K}$, et al. Posttranslational modifications mediate the structural diversity of tauopathy strains. Cell. (2020) 180:633-44.e12. doi: 10.1016/j.cell.2020.01.027

9. Zhang W, Tarutani A, Newell KL, Murzin AG, Matsubara T, Falcon B, et al. Novel tau filament fold in corticobasal degeneration. Nature. (2020) 580:283-7. doi: 10.1101/811703

10. Swatek KN, Komander D. Ubiquitin modifications. Cell Res. (2016) 26:399422. doi: $10.1038 / \mathrm{cr} .2016 .39$

11. Galves M, Rathi R, Prag G, Ashkenazi A. Ubiquitin signaling and degradation of aggregate-prone proteins. Trends Biochem Sci. (2019) 44:87284. doi: 10.1016/j.tibs.2019.04.007

12. Dittmar G, Selbach M. Deciphering the ubiquitin code. Mol Cell. (2017) 65:779-80. doi: 10.1016/j.molcel.2017.02.011

13. Grumati P, Dikic I. Ubiquitin signaling and autophagy. J Biol Chem. (2018) 293:5404-13. doi: 10.1074/jbc.TM117.000117

14. Levine B, Kroemer G. Autophagy in the pathogenesis of disease. Cell. (2008) 132:27-42. doi: 10.1016/j.cell.2007.12.018

15. Ukmar-Godec T, Hutten S, Grieshop MP, Rezaei-Ghaleh N, Cima-Omori MS, Biernat J, et al. Lysine/RNA -interactions drive and regulate biomolecular condensation. Nat Commun. (2019) 10:2909. doi: 10.1038/s41467-019-10792-y

16. Lee G, Newman ST, Gard DL, Band H, Panchamoorthy G. Tau interacts with src-family non-receptor tyrosine kinases. J Cell Science. (1998) 111 (Pt 21):3167-77. doi: 10.1242/jcs.111.21.3167

17. Lee G, Thangavel R, Sharma VM, Litersky JM, Bhaskar K, Fang SM, et al. Phosphorylation of tau by fyn: implications for Alzheimer's disease. J Neurosci. (2004) 24:2304-12. doi: 10.1523/JNEUROSCI.4162-03.2004

18. Wang Y, Mandelkow E. Tau in physiology and pathology. Nat Rev Neurosci. (2016) 17:5-21. doi: 10.1038/nrn.2015.1

19. Morishima-Kawashima M, Hasegawa M, Takio K, Suzuki M, Titani K, Ihara Y. Ubiquitin is conjugated with amino-terminally processed tau in paired helical filaments. Neuron. (1993) 10:1151-60. doi: 10.1016/0896-6273(93)90063-W

20. Cripps D, Thomas SN, Jeng Y, Yang F, Davies P, Yang AJ. Alzheimer diseasespecific conformation of hyperphosphorylated paired helical filament-Tau is polyubiquitinated through Lys-48, Lys-11, and Lys-6 ubiquitin conjugation. $J$ Biol Chem. (2006) 281:10825-38. doi: 10.1074/jbc.M512786200

21. Nakayama Y, Sakamoto S, Tsuji K, Ayaki T, Tokunaga F, Ito H. Identification of linear polyubiquitin chain immunoreactivity in tau pathology of Alzheimer's disease. Neurosci Lett. (2019) 703:53-7. doi: 10.1016/j.neulet.2019.03.017

22. Tan JM, Wong ES, Kirkpatrick DS, Pletnikova O, Ko HS, Tay SP, et al. Lysine 63-linked ubiquitination promotes the formation and autophagic clearance of protein inclusions associated with neurodegenerative diseases. $\mathrm{Hum} \mathrm{Mol}$ Genet. (2008) 17:431-9. doi: 10.1093/hmg/ddm320

23. Liu A, Gong P, Hyun SW, Wang KZ, Cates EA, Perkins D, et al. TRAF6 protein couples Toll-like receptor 4 signaling to Src family kinase activation and opening of paracellular pathway in human lung microvascular endothelia. J Biol Chem. (2012) 287:16132-45. doi: 10.1074/jbc.M111.310102

24. Brickner JR, Soll JM, Lombardi PM, Vågbø CB, Mu dge MC, Oyeniran C, et al. A ubiquitin-dependent signalling axis specific for ALKBH-mediated DNA dealkylation repair. Nature. (2017) 551:389-93. doi: 10.1038/nature24484

25. Babu JR, Geetha T, Wooten MW. Sequestosome 1/p62 shuttles polyubiquitinated tau for proteasomal degradation. J Neurochem. (2005) 94:192-203. doi: 10.1111/j.1471-4159.2005.03181.x

26. Nakazawa S, Oikawa D, Ishii R, Ayaki T, Takahashi H, Takeda $\mathrm{H}$, et al. Linear ubiquitination is involved in the pathogenesis of optineurin-associated amyotrophic lateral sclerosis. Nat Commun. (2016) 7:12547. doi: $10.1038 /$ ncomms 12547

27. Munari F, Barracchia CG, Franchin C, Parolini F, Capaldi S, Romeo A, et al. Semisynthetic and enzyme-mediated conjugate preparations illuminate the ubiquitination-dependent aggregation of tau protein. Angewandte Chemie (International ed. in English). (2020) 59:6607-11. doi: 10.1002/anie.201916756

28. Ye Y, Klenerman D, Finley D. N-Terminal ubiquitination of amyloidogenic proteins triggers removal of their oligomers by the proteasome holoenzyme. $J$ Mol Biol. (2020) 432:585-96. doi: 10.1016/j.jmb.2019.08.021

29. Flach K, Ramminger E, Hilbrich I, Arsalan-Werner A, Albrecht F, Herrmann $\mathrm{L}$, et al. Axotrophin/MARCH7 acts as an E3 ubiquitin ligase and ubiquitinates tau protein in vitro impairing microtubule binding. Biochimica Biophysica Acta. (2014) 1842:1527-38. doi: 10.1016/j.bbadis.2014.05.029

30. Tatham MH, Plechanovová A, Jaffray EG, Salmen H, Hay RT. Ube2W conjugates ubiquitin to $\alpha$-amino groups of protein $\mathrm{N}$-termini. Biochem J. (2013) 453:137-45. doi: 10.1042/BJ20130244

31. Petrucelli L, Dickson D, Kehoe K, Taylor J, Snyder H, Grover A, et al. CHIP and Hsp70 regulate tau ubiquitination, degradation and aggregation. Hum Mol Genet. (2004) 13:703-14. doi: 10.1093/hmg/ddh083

32. Seibenhener ML, Babu JR, Geetha T, Wong HC, Krishna NR, Wooten MW. Sequestosome 1/p62 is a polyubiquitin chain binding protein involved in ubiquitin proteasome degradation. Mol Cell Biol. (2004) 24:805568. doi: 10.1128/MCB.24.18.8055-8068.2004

33. Subramanian M, Hyeon SJ, Das T, Suh YS, Kim YK, Lee JS, et al. UBE4B, a microRNA-9 target gene, promotes autophagy-mediated Tau degradation. Nat Commun. (2021) 12:3291. doi: 10.1038/s41467-021-23597-9

34. Obin M, Mesco E, Gong X, Haas AL, Joseph J, Taylor A. Neurite outgrowth in PC12 cells. Distinguishing the roles of ubiquitylation and ubiquitin-dependent proteolysis. J Biol Chem. (1999) 274:1178995. doi: $10.1074 /$ jbc. 274.17 .11789

35. Goedert M. Tau protein and neurodegeneration. Semin Cell Dev Biol. (2004) 15:45-9. doi: 10.1016/j.semcdb.2003.12.015

36. S. Jeganathan, von Bergen $M$, Brutlach $H$, Steinhoff HJ, Mandelkow E. Global hairpin folding of tau in solution. Biochemistry. (2006) 45:228393. doi: 10.1021/bi0521543

37. Iqbal K, Liu F, Gong CX. Tau and neurodegenerative disease: the story so far. Nat Rev Neurol. (2016) 12:15-27. doi: 10.1038/nrneurol.2015.225

38. Wesseling H, Mair W, Kumar M, Schlaffner CN, Tang S, Beerepoot P, et al. Tau PTM profiles identify patient heterogeneity and stages of Alzheimer's disease. Cell. (2020) 183:1699-713.e13. doi: 10.1016/j.cell.2020.10.029

39. Dujardin S, Commins C, Lathuiliere A, Beerepoot P, Fernandes AR, Kamath TV, et al. Tau molecular diversity contributes to clinical heterogeneity in Alzheimer's disease. Nat Med. (2020) 26:1256-63. doi: 10.1038/s41591-020-0938-9

40. Long JM, Holtzman DM. Alzheimer disease: an update on pathobiology and treatment strategies. Cell. (2019) 179:312-39. doi: 10.1016/j.cell.2019.09.001

41. Barthelemy NR, Bateman RJ, Hirtz C, Marin P, Becher F, Sato C, et al. Alzheimer's Res Ther. (2020) 12:26. doi: 10.1186/s13195-020-00596-4

42. Janelidze S, Mattsson N, Palmqvist S, Smith R, Beach TG, Serrano GE, et al. Plasma P-tau181 in Alzheimer's disease: relationship to other biomarkers, differential diagnosis, neuropathology and longitudinal progression to Alzheimer's dementia. Nat Med. (2020) 26:379-86. doi: 10.1038/s41591-020-0755-1 
43. Braak H, Braak E, Staging of Alzheimer's disease-related neurofibrillary changes. Neurobiol Aging. (1995) 16:271-8; discussion 278-84. doi: 10.1016/0197-4580(95)00021-6

44. Cente M, Filipcik P, Pevalova M, Novak M. Expression of a truncated tau protein induces oxidative stress in a rodent model of tauopathy. Eur J Neurosci. (2006) 24:1085-90. doi: 10.1111/j.1460-9568.2006.04986.x

45. Vanderweyde T, Apicco DJ, Youmans-Kidder K, Ash PEA, Cook C, Lummertz da Rocha E, et al. Interaction of tau with the RNA-Binding Protein TIA1 regulates tau pathophysiology and toxicity. Cell Rep. (2016) 15:145566. doi: 10.1016/j.celrep.2016.04.045

46. Berry RW, Abraha A, Lagalwar S, LaPointe N, Gamblin TC, Cryns VL, et al. Inhibition of tau polymerization by its carboxy-terminal caspase cleavage fragment. Biochemistry. (2003) 42:8325-31. doi: 10.1021/bi027348m

47. Gamblin TC, Berry RW, Binder LI. Tau polymerization: role of the amino terminus. Biochemistry. (2003) 42:2252-7. doi: 10.1021/bi0272510

48. Bancher C, Grundke-Iqbal I, Iqbal K, Fried VA, Smith HT, Wisniewski HM. Abnormal phosphorylation of tau precedes ubiquitination in neurofibrillary pathology of Alzheimer disease. Brain Res. (1991) 539:11-8. doi: 10.1016/0006-8993(91)90681-K

49. Bancher C, Brunner C, Lassmann H, Budka H, Jellinger K, Seitelberger F, et al. Tau and ubiquitin immunoreactivity at different stages of formation of Alzheimer neurofibrillary tangles. Prog Clin Biol Res. (1989) 317:837-48.

50. Lasagna-Reeves CA, Castillo-Carranza DL, Sengupta U, Sarmiento J, Troncoso J, Jackson GR, et al. Identification of oligomers at early stages of tau aggregation in Alzheimer's disease. FASEB J. (2012) 26:194659. doi: 10.1096/fj.11-199851

51. Iqbal K, Gong CX, Liu F. Hyperphosphorylation-induced tau oligomers. Front Neurol. (2013) 4:112. doi: 10.3389/fneur.2013.00112

52. Park S, Lee JH, Jeon JH, Lee MJ. Degradation or aggregation: the ramifications of post-translational modifications on tau. BMB Rep. (2018) 51:26573. doi: 10.5483/BMBRep.2018.51.6.077

53. Dickey CA, Yue M, Lin WL, Dickson DW, Dunmore JH, Lee WC, et al. Deletion of the ubiquitin ligase CHIP leads to the accumulation, but not the aggregation, of both endogenous phospho- and caspase-3-cleaved tau species. J Neurosci. (2006) 26:6985-96. doi: 10.1523/JNEUROSCI.0746-06.2006

54. Cliffe R, Sang JC, Kundel F, Finley D, Klenerman D, Ye Y. Filamentous aggregates are fragmented by the proteasome holoenzyme. Cell Rep. (2019) 26:2140-9.e3. doi: 10.1016/j.celrep.2019.01.096

55. Myeku N, Clelland CL, Emrani S, Kukushkin NV, Yu WH, Goldberg AL, et al. Tau-driven $26 \mathrm{~S}$ proteasome impairment and cognitive dysfunction can be prevented early in disease by activating cAMP-PKA signaling. Nat Med. (2016) 22:46-53. doi: 10.1038/nm.4011

56. Keller JN, Hanni KB, Markesbery WR. Impaired proteasome function in Alzheimer's disease. J Neurochem. (2000) 75:4369. doi: 10.1046/j.1471-4159.2000.0750436.x

57. Santacruz K, Lewis J, Spires T, Paulson J, Kotilinek L, Ingelsson M, et al. Tau suppression in a neurodegenerative mouse model improves memory function. Science. (2005) 309:476-81. doi: 10.1126/science.1113694

58. Ghag G, Bhatt N, Cantu DV, Guerrero-Munoz MJ, Ellsworth A, Sengupta $\mathrm{U}$, et al. Soluble tau aggregates, not large fibrils, are the toxic species that display seeding and cross-seeding behavior. Protein Sci. (2018) 27:19019. doi: $10.1002 /$ pro.3499
59. Balaji V, Kaniyappan S, Mandelkow E, Wang Y, Mandelkow EM. Pathological missorting of endogenous MAPT/Tau in neurons caused by failure of protein degradation systems. Autophagy. (2018) 14:213954. doi: 10.1080/15548627.2018.1509607

60. Jackson SJ, Kerridge C, Cooper J, Cavallini A, Falcon B, Cella CV, et al. Short fibrils constitute the major species of seed-competent tau in the brains of mice transgenic for human P301S tau. J Neurosci. (2016) 36:76272. doi: 10.1523/JNEUROSCI.3542-15.2016

61. Takeda S, Wegmann S, Cho H, DeVos SL, Commins C, Roe AD, et al. Neuronal uptake and propagation of a rare phosphorylated high-molecularweight tau derived from Alzheimer's disease brain. Nat Commun. (2015) 6:8490. doi: 10.1038/ncomms9490

62. Li L, Shi R, Gu J, Tung YC, Zhou Y, Zhou D, et al. Alzheimer's disease brain contains tau fractions with differential prion-like activities. Acta Neuropathol Commun. (2021) 9:28. doi: 10.1186/s40478-021-01127-4

63. Mirbaha H, Holmes BB, Sanders DW, Bieschke J, Diamond MI. Tau trimers are the minimal propagation unit spontaneously internalized to seed intracellular aggregation. J Biol Chem. (2015) 290:14893-903. doi: 10.1074/jbc.M115.652693

64. Morsch R, Simon W, Coleman PD. Neurons may live for decades with neurofibrillary tangles. J Neuropathol Exp Neurol. (1999) 58:18897. doi: 10.1097/00005072-199902000-00008

65. de Calignon, Spires-Jones TL, Pitstick R, Carlson GA, Hyman BT. Tanglebearing neurons survive despite disruption of membrane integrity in a mouse model of tauopathy. J Neuropathol Exp Neurol. (2009) 68:75761. doi: 10.1097/NEN.0b013e3181a9fc66

66. Chu TT, Gao N, Li QQ, Chen PG, Yang XF, Chen YX, et al. Specific knockdown of endogenous tau protein by peptidedirected ubiquitin-proteasome degradation. Cell Chem Biol. (2016) 23:453-61. doi: 10.1016/j.chembiol.2016.02.016

67. Wang W, Zhou Q, Jiang T, Li S, Ye J, Zheng J, et al. A novel small-molecule PROTAC selectively promotes tau clearance to improve cognitive functions in Alzheimer-like models. Theranostics. (2021) 11:527995. doi: 10.7150/thno. 55680

Conflict of Interest: The authors declare that the research was conducted in the absence of any commercial or financial relationships that could be construed as a potential conflict of interest.

Publisher's Note: All claims expressed in this article are solely those of the authors and do not necessarily represent those of their affiliated organizations, or those of the publisher, the editors and the reviewers. Any product that may be evaluated in this article, or claim that may be made by its manufacturer, is not guaranteed or endorsed by the publisher.

Copyright (C) 2022 Li, Jiang, Wang, Liu and Wang. This is an open-access article distributed under the terms of the Creative Commons Attribution License (CC BY). The use, distribution or reproduction in other forums is permitted, provided the original author(s) and the copyright owner(s) are credited and that the original publication in this journal is cited, in accordance with accepted academic practice. No use, distribution or reproduction is permitted which does not comply with these terms. 